\title{
Seletividade de coroa-de-cristo (Euphorbia splendens) a diferentes classes de herbicidas para manejo de plantas daninhas em jardinagem
}

\author{
CLEBER D. DE G. MACIEL ${ }^{1}$; JULIANAP. POLETINE ${ }^{1}$; JAMIL CONSTANTIN ${ }^{2}$; CELSO E. JARDIM ${ }^{3}$; \\ RODRIGO DOS S. BERNANDO ${ }^{3}$; ERNESTO R. MOUTA ${ }^{3}$ e DÊNIS R. S. BELISÁRIO ${ }^{3}$
}

\begin{abstract}
RESUMO
Com objetivo de avaliar a seletividade da coroa-de-cristo (Euphorbia splendes) para diferentes classes de herbicidas, três experimentos foram realizados, em condições de campo, na Fazenda Modelo da ESAPP/SP (2001/2002). Os tratamentos do Experimento 1 foram (em e.a. ou i.a.): glyphosate (1,44 kg.ha $\left.{ }^{-1}\right)$; sulfosate (1,44 kg.ha $\left.{ }^{-1}\right)$; amônio-glufosinato $\left(0,48 \mathrm{~kg} \cdot \mathrm{ha}^{-1}\right)$; paraquat $\left(0,60 \mathrm{~kg} \cdot \mathrm{ha}^{-1}\right)$; glyphosate + flumioxazin $\left(1,44+0,06 \mathrm{~kg} \cdot \mathrm{ha}^{-}\right.$ $\left.{ }^{1}\right)$; glyphosate + carfentrazone-ethyl $\left(1,44+0,06 \mathrm{~kg} \cdot \mathrm{ha}^{-1}\right)$ e testemunha. No Experimento 2: carfentrazone-ethyl $\left(0,12 \mathrm{~kg} \cdot \mathrm{ha}^{-1}\right) ; 2,4-\mathrm{D}\left(1,30 \mathrm{~kg} \cdot \mathrm{ha}^{-1}\right)$; isoxaflutole $\left(0,06 \mathrm{~kg} \cdot \mathrm{ha}^{-1}\right)$; nicosulfuron $\left(0,06 \mathrm{~kg} \cdot \mathrm{ha}^{-1}\right)$; metsulfuron-methyl $\left(0,0024 \mathrm{~kg} \cdot \mathrm{ha}^{-1}\right)$; lactofen $\left(0,180 \mathrm{~kg} \cdot \mathrm{ha}^{-1}\right)$; chlorimuron-ethyl $\left(0,02 \mathrm{~kg} \cdot \mathrm{ha}^{-1}\right)$; bentazon $\left(1,25 \mathrm{~kg} \cdot \mathrm{ha}^{-1}\right)$ e testemunha. No Experimento 3 foram testados: fluazifop-p-butyl $\left(0,25 \mathrm{~kg} \cdot \mathrm{ha}^{-1}\right)$; sethoxydim $\left(1,25 \mathrm{~kg} \cdot \mathrm{ha}^{-1}\right)$; haloxyfop-methyl $\left(0,06 \mathrm{~kg} \cdot \mathrm{ha}^{-1}\right)$; clethodim + fenoxaprop-p-ethyl $\left(0,11 \mathrm{~kg} \cdot \mathrm{ha}^{-1}\right)$; quizalofop-p-ethyl $\left(0,10 \mathrm{~kg} \mathrm{ha}^{-1}\right)$; clethodim $(0,12$ kg.ha- $\left.{ }^{-1}\right)$; propaquizafop $\left(0,12 \mathrm{~kg} \cdot \mathrm{ha}^{-1}\right)$; tepraloxydim $\left(0,40 \mathrm{~kg} \cdot \mathrm{ha}^{-1}\right)$; butroxydim $\left(0,10 \mathrm{~kg} \cdot \mathrm{ha}^{-1}\right)$ e testemunha. No Experimento 1, nenhum tratamento foi seletivo, causando danos visíveis de fitointoxicação a partir dos 7 dias após aplicação. No Experimento 2, apenas o metsulfuron-methyl apresentou seletividade à coroa-de-cristo, para as folhas e inflorescências; sendo o isoxaflutole, nicosulfuron, chlorimuron-ethyl e bentazon impróprios apenas no que diz respeito à preservação de flores. No Experimento 3, todos os tratamentos indicaram seletividade à coroa-de-cristo, observando-se apenas injúrias às flores para os herbicidas fluazifop-p-butil e sethoxydim e propaquizafop.
\end{abstract}

Palavras-chave: fitointoxicação, pós-emergência, plantas ornamentais e mecanismo de ação.

\begin{abstract}
Selectivity of Euphorbia splendens to distinct herbicide classes in gardening weed management. In order to evaluate the selectivity of Euphorbia splendens to different kinds of herbicides, three tests were developed in the experimental area of ESAPP/SP Model Farm (2001/2002). The treatments of the experiment 1 were (in e.a. or i.a.): glyphosate $\left(1,44 \mathrm{~kg} \cdot \mathrm{ha}^{-1}\right)$; sulfosate $\left(1,44 \mathrm{~kg} \cdot \mathrm{ha}^{-1}\right)$; ammonium-gluphosinate $\left(0,48 \mathrm{~kg} \cdot \mathrm{ha}^{-1}\right)$; paraquat $(0,60$ kg.ha-1 $)$; glyphosate + flumioxazin $\left(1,44+0,06 \mathrm{~kg} \cdot \mathrm{ha}^{-1}\right)$; glyphosate + carfentrazone-ethyl $\left(1,44+0,06 \mathrm{~kg} \cdot \mathrm{ha}^{-1}\right)$ and one check. In the experiment 2: carfentrazone-ethyl $\left(0,12 \mathrm{~kg}^{-h^{-1}}\right) ; 2,4-\mathrm{D}\left(1,30 \mathrm{~kg}^{-h \mathrm{~h}^{-1}}\right)$; isoxaflutole $(0,06$ kg.ha $\left.{ }^{-1}\right)$; nicosulfuron $\left(0,06 \mathrm{~kg} \cdot \mathrm{ha}^{-1}\right)$; metsulfuron-methyl $\left(0,0024 \mathrm{~kg}_{\text {.ha }}{ }^{-1}\right)$; lactofen $\left(0,180 \mathrm{~kg} \cdot \mathrm{ha}^{-1}\right)$; chlorimuronethyl $\left(0,02 \mathrm{~kg} \cdot \mathrm{ha}^{-1}\right)$; bentazon $\left(1,25 \mathrm{~kg} \cdot \mathrm{ha}^{-1}\right)$ and one check. In the third experiment were tested: fluazifop-p-butyl $\left(0,25 \mathrm{~kg} \cdot \mathrm{ha}^{-1}\right)$; sethoxydim $\left(1,25 \mathrm{~kg} \cdot \mathrm{ha}^{-1}\right)$; haloxyfop-methyl $\left(0,06 \mathrm{~kg} \cdot \mathrm{ha}^{-1}\right)$; clethodim + fenoxaprop-p-ethyl $(0,11$ kg.ha-1 $)$; quizalofop-p-ethyl $\left(0,10 \mathrm{~kg} \cdot \mathrm{ha}^{-1}\right)$; clethodim $\left(0,12 \mathrm{~kg} \cdot \mathrm{ha}^{-1}\right)$; propaquizafop $\left(0,12 \mathrm{~kg} \cdot \mathrm{ha}^{-1}\right)$; tepraloxydim $\left(0,40 \mathrm{~kg} \cdot \mathrm{ha}^{-1}\right)$; butroxydim $\left(0,10 \mathrm{~kg} \cdot \mathrm{ha}^{-1}\right)$ and one check. In the experiment 1 , none of the treatments were selective, causing visible phytointoxication damages starting from 7 days after application. In the Experiment 2, only metsulfuron-methyl presented selectivity to E. splendens, for leaves and inflorescences levels, being isoxaflutole, nicosulfuron, chlorimuron-ethyl and bentazon inappropriate treatments just for flowers preservation. In the third experiment, all treatments showed selectivity to $E$. splendens, occuring damages on inflorescences level only for fluazifop-p-butil, sethoxydim and propaquizafop herbicides.
\end{abstract}

Key words: fitotoxicity, post-emergency, ornamental plants e mechanism of action.

\section{INTRODUÇÃO}

A coroa-de-cristo (Euphorbia splendens), também popularmente conhecida como "dois irmãos" ou "bemcasados", é uma planta arbustiva da família das Euforbiáceas, sendo constituída por caule escandente e ramos espinhentos, tortuosos e prostrados que dão ao conjunto um aspecto de moita. Suas folhas, de coloração verde-opaca, são pequenas e encontram-se dispostas somente nas pontas dos caules, sendo quase ausentes em condição de inverno seco, ao contrário de suas flores, as quais se encontram presentes aos pares e sustentadas por duas brácteas vermelhas em todas as estações do ano (BIACHINI \& PANTANO, 1994).

Rústica, a coroa-de-cristo é tipicamente considerada uma planta que se adapta a lugares secos $3 / 4$ haja vista sua

\footnotetext{
${ }^{1}$ Prof. Dr. Departamento de Ciências Biológicas e Fitossanitárias, ESAPP, 19700-000, Paraguaçu Paulista, SP.

2 Prof. Dr. Departamento de Agronomia, UEM, 87020-900, Maringá, PR.

${ }^{3}$ Alunos do Curso de Agronomia, ESAPP, 19700-000, Paraguaçu Paulista, SP.
} 
origem em Madagascar $3 / 4$ o que facilita sua multiplicação por meio de estacas, e pode atingir até dois metros de comprimento. A utilização da espécie Euphorbia splendens é amplamente difundida como cerca viva, uma excelente barreira física na decoração de ambientes urbanos, pode ser encontrada facilmente na entrada de cidades, em praças e canteiros centrais de avenidas, porque permite podas em formatos diversos (ZIMBER, 1946; LORENZI \& SOUZA, 2001; RURALNEWS, 2004). Entretanto, apesar de sua grande versatilidade decorativa, ao ser cultivada em diferentes ambientes, o manejo dessa variedade em relação ao controle de plantas daninhas e/ou parasitas, tais como o cipó-chumbo (Cuscuta racemosa Mart.), que se caracteriza por formar um manto de filamentos dourados sobre as folhagens de espécies ornamentais (LORENZI, 2000), tem sido realizado por meio de capinas ou limpeza manual, quando o adensamento das plantas é maior.

$\mathrm{O}$ desconhecimento e/ou inexistência de informações técnicas, assim como de trabalhos publicados referentes à seletividade dos diferentes herbicidas encontrados no mercado para plantas ornamentais, têm limitado a maior versatilidade do manejo de plantas daninhas e/ou parasitas em ambientes decorados com espécies, tais como a coroa-de-cristo. Nesse contexto, dentre as práticas de jardinagem, necessárias para manutenção de sua beleza visual, o controle químico pode tornar-se uma alternativa viável, uma vez que haveria economia considerável sob o ponto de vista de utilização de mão-de-obra e tempo de operação.

Dessa forma, o trabalho teve como objetivo avaliar a seletividade da coroa-de-cristo (E. splendens) a diferentes classes de herbicidas de uso agrícola, considerando a preservação de aspectos de jardinagem da espécie.

\section{MATERIALEMÉTODOS}

Entre os anos de 2001 e 2002, três experimentos foram realizados em condições de campo na Fazenda Modelo, pertencente à Escola Superior de Agronomia de Paraguaçu Paulista - ESAPP, localizada no Município de Paraguaçu Paulista, SP (Médio Vale do Paranapanema) a $22^{\circ} 24^{\prime} 46^{\prime \prime}$ de latitude Sul e 50 34' 33" de longitude Oeste, em altitude de $480 \mathrm{~m}$ (IBGE-CIDADES, 2004).

As unidades experimentais utilizadas foram representadas por vasos de cimento com $40 \mathrm{~cm}$ de diâmetro e $70 \mathrm{~cm}$ de altura, onde, em 10 de dezembro de 2001, foram plantadas a $8 \mathrm{~cm}$ de profundidade, três estacas apicais de coroa-de-cristo (E. splendens) com $35 \mathrm{~cm}$ de comprimento e algumas folhas no terço médio apical. As estacas foram cortadas a partir do ápice de ramos saudáveis de plantas adultas em pleno desenvolvimento, com seleção prévia de sua espessura, e após serem cortadas das plantas mãe e lavadas em água morna para estancar a seiva, ainda foram secadas em condições de sombreamento e aeração durante três dias, antes de serem plantadas nos vasos, conforme recomendações de BLOSSFELD (1965) e ZIMBER (1946).

O substrato utilizado foi representado por uma mistura homogênea de quatro partes de solo retirado da camada arável e classificado como Latossolo distroférico (textura arenosa), para uma parte de composto orgânico constituído por estercos de gado e de galinha, adicionando- se à mistura 20 g.vaso ${ }^{-1}$ da fórmula 4-14-8.

Em todos experimentos foi adotado o delineamento experimental de blocos ao acaso, com cinco repetições. Os tratamentos do Experimento 1 foram constituídos por herbicidas e misturas, classificados como de produtos de ação total (não seletivos): glyphosate (1,44 kg e.a.ha$\left.{ }^{1}\right)$; sulfosate (1,44 kg e.a.ha-1 $)$; amônio-gluphosinato (0,48 kg i.a.ha $\left.{ }^{-1}\right)$; paraquat $\left(0,60 \mathrm{~kg}\right.$ i.a.ha $\left.{ }^{-1}\right)$; glyphosate + flumioxazin $\left(1,44 \mathrm{~kg}\right.$ e.a.ha-1 $+0,06 \mathrm{~kg}$ i.a.ha $\left.{ }^{-1}\right)$; glyphosate + carfentrazone-ethyl (1,44 kg e.a. ha ${ }^{-1}+0,06 \mathrm{~kg}$ i.a.ha $\left.{ }^{-1}\right)$ e testemunha. No Experimento 2 utilizaram-se herbicidas "latifolicidas" (não seletivos para algumas espécies dicotiledôneas): carfentrazone-ethyl (0,12 kg i.a.ha-1 $)$; 2,4D $\left(1,30 \mathrm{~kg}\right.$ e.a.ha $\left.{ }^{-1}\right)$; isoxaflutole $(0,06 \mathrm{~kg}$ e.a.ha-1 $)$; nicosulfuron $\left(0,06 \mathrm{~kg}\right.$ i.a.ha $\left.{ }^{-1}\right)$; metsulfuron-methyl $(0,0024$ kg i.a.ha-1 $)$; lactofen $\left(0,180 \mathrm{~kg}\right.$ i.a.ha $\left.{ }^{-1}\right)$; chlorimuron-ethyl $(0,02 \mathrm{~kg}$ i.a.ha-1 $)$; bentazon $\left(1,25 \mathrm{~kg}\right.$ i.a.ha $\left.{ }^{-1}\right)$ e testemunha. No Experimento 3 foram testados herbicidas "graminicidas" (não seletivos para as espécies monocotiledôneas): fluazifop-p-butil $\left(0,25 \mathrm{~kg}\right.$ i.a.ha- $\left.{ }^{-1}\right)$; sethoxydim $(1,25 \mathrm{~kg}$ i.a.ha-1 $)$; haloxyfop-methyl $(0,06 \mathrm{~kg}$ i.a.ha-1 ${ }^{-1}$; clethodim + fenoxaprop-p-ethyl $(0,11 \mathrm{~kg}$ i.a.ha$\left.{ }^{1}\right)$; quizalofop-p-ethyl (0,10 kg i.a.ha-1 $)$; clethodim $(0,12$ kg i.a.ha- $\left.{ }^{-1}\right)$; propaquizafop $\left(0,12 \mathrm{~kg}\right.$ i.a.ha $\left.{ }^{-1}\right)$; tepraloxydim $\left(0,40 \mathrm{~kg}\right.$ i.a.ha $\left.{ }^{-1}\right)$; butroxydim $\left(0,10 \mathrm{~kg}\right.$ i.a.ha $\left.{ }^{-1}\right) \mathrm{e}$ testemunha. Para o herbicida paraquat foi adicionado na solução de aplicação o adjuvante Agralâ na dose de 0,2 $\%$ v/v (volume/volume), mesmo para metsulfuron-methyl, chlorimuron-ethyl e bentazon. Para os produtos do Experimento 3 utilizou-se Assistâ a 0,5\% v/v.

As aplicações dos herbicidas nos três experimentos foram efetuadas em pós-emergência no final da tarde de 9 de novembro de 2002, com as plantas de coroa-de-cristo em início de florescimento e o solo úmido, utilizou-se pulverizador costal a pressão constante de $2,1 \mathrm{kgf} . \mathrm{cm}^{-2}$ mantida pelo $\mathrm{CO}_{2}$ pressurizado com quatro pontas XR11002-VS, e consumo de calda de 200 L.ha $^{-1}$. No momento das aplicações dos tratamentos as condições climáticas encontravam-se com temperaturas de $24,4^{\circ} \mathrm{C}$; $22,7{ }^{\circ} \mathrm{Ce} 22,0^{\circ} \mathrm{C}$, umidade relativa do ar de $64,0 \% ; 68,5 \%$ $68,9 \%$ e ventos com rajadas de $1,4 \mathrm{~km} \cdot \mathrm{h}^{-1} ; 2,0 \mathrm{~km} \cdot \mathrm{h}^{-1}$ e 1,6 $\mathrm{km} \cdot \mathrm{h}^{-1}$, respectivamente para os Experimentos 1, 2 e 3.

As características analisadas foram constituídas pela porcentagem de fitointoxicação visual aos 7, 14, 21, 28 e 35 dias após aplicação (DAA), considerando injúrias nas folhas, flores e caule, onde " $0 \%$ " corresponde à "ausência de danos" e "100\%" à "morte das plantas", conforme procedimentos da E.W.R.C. (1964) e da Sociedade Brasileira da Ciência das Plantas Daninhas (1995), assim como do teor de clorofila (índice SPAD) da parte mediana do limbo foliar, avaliado em folhas da região apical das plantas aos 14, 21, 28 e 35 DAA, utilizando-se clorofilômetro portátil modelo MINOLTA 502.

Os dados obtidos foram submetidos à análise de variância pelo teste $\mathrm{F}$ e suas médias comparadas pelo teste de Tukey a $5 \%$ de probabilidade.

\section{RESULTADOS E DISCUSSÃO}

No Experimento 1 nenhum tratamento apresentou seletividade aceitável, ocorrendo danos visíveis de 
fitointoxicação superiores a 37,4\% aos 35 dias após aplicação (DAA), o que levou à queda parcial e/ou total das folhas e flores, assim como necrosamento de folhas e paralisação do desenvolvimento dos ápices das plantas (Tabela 1). A partir dos 7 DAA, os herbicidas paraquat $\left(0,60\right.$ kg.ha $\left.{ }^{-1}\right)$, ghyphosate + carfentrazone-ethyl $(1,44+$
0,06 kg.ha-1 $)$, amônio-glufosinato $\left(0,48 \mathrm{~kg} \cdot \mathrm{ha}^{-1}\right)$ e glyphosate + flumioxazin $\left(1,44+0,06 \mathrm{~kg} \cdot \mathrm{ha}^{-1}\right)$ apresentaram maior velocidade de ação fitotóxica para coroa-de-cristo $(E$. splendens), ocasionando estragos mais intensos nas flores, folhas e ápices das plantas, quando comparado ao glyphosate $\left(1,44 \mathrm{~kg} \cdot \mathrm{ha}^{-1}\right)$ e sulfosate $\left(1,44 \mathrm{~kg} \cdot \mathrm{ha}^{-1}\right)$ aplicados isoladamente. (Tabela 1)

Tabela 1. Porcentagem de fitointoxicação na parte aérea e teor de clorofila nas folhas das plantas de coroa-de-cristo, submetidas a aplicações de herbicidas classificados como de "ação total” (Experimento 1). ESAPP/Paraguaçu PaulistaSP, 2001/2002.

\begin{tabular}{|c|c|c|c|c|c|c|c|c|c|c|}
\hline \multirow[t]{2}{*}{ Tratamentos } & \multirow{2}{*}{$\begin{array}{c}\text { Doses } \\
\text { kg e.a. ou } \\
\text { i.a. ha-1 }\end{array}$} & \multicolumn{5}{|c|}{ Fitointoxicação (\%) } & \multicolumn{4}{|c|}{ Teor de Clorofila $\left(\mu \mathrm{g} \mathrm{dm^{-2 } )}\right.$} \\
\hline & & $\begin{array}{c}7 \\
\text { DAA }^{/ 1}\end{array}$ & $\begin{array}{c}14 \\
\text { DAA }\end{array}$ & $\begin{array}{c}21 \\
\text { DAA }\end{array}$ & $\begin{array}{c}28 \\
\text { DAA }\end{array}$ & $\begin{array}{c}35 \\
\text { DAA }\end{array}$ & $\begin{array}{c}14 \\
\text { DAA }\end{array}$ & $\begin{array}{c}21 \\
\text { DAA }\end{array}$ & $\begin{array}{c}28 \\
\text { DAA }\end{array}$ & 35 DAA \\
\hline T1) glyphosate & 1,44 & $16,2 \mathrm{~d}$ & $37,6 \mathrm{c}$ & $34,2 \mathrm{~d}$ & $30,8 \mathrm{c}$ & $40,2 \mathrm{bc}$ & $54,8 \mathrm{a}$ & $61,2 \mathrm{ab}$ & $68,7 \mathrm{a}$ & $68,7 \mathrm{a}$ \\
\hline T2) sulfosate & 1,44 & $15,6 \mathrm{~d}$ & $30,6 \mathrm{c}$ & $25,4 \mathrm{~d}$ & $33,4 \mathrm{c}$ & $37,4 \mathrm{c}$ & 55,9 a & $59,5 \mathrm{ab}$ & 67,4 a & $67,4 \mathrm{a}$ \\
\hline $\begin{array}{l}\text { T3) amônio- } \\
\text { glufosinato }\end{array}$ & 1,44 & $78,6 \mathrm{~b}$ & 89,0 a & $75,6 \mathrm{~b}$ & $68,8 \mathrm{~b}$ & $56,0 \mathrm{bc}$ & $0,0 \mathrm{~b}$ & $39,3 \mathrm{bc}$ & $58,1 \mathrm{~b}$ & $60,1 \mathrm{ab}$ \\
\hline T4) paraquat & 0,60 & 95,8 a & 96,0 a & $97,0 \mathrm{a}$ & 97,4 a & $96,0 \mathrm{a}$ & $0,0 \mathrm{~b}$ & $17,1 \mathrm{~cd}$ & $0,0 \mathrm{c}$ & $0,0 \mathrm{c}$ \\
\hline $\begin{array}{l}\text { T5) glyphosate + } \\
\text { flumioxazin }\end{array}$ & $\begin{array}{c}1,44+ \\
0,06\end{array}$ & $36,2 \mathrm{c}$ & $68,6 \mathrm{~b}$ & $60,0 \mathrm{c}$ & $62,6 \mathrm{~b}$ & $59,0 \mathrm{~b}$ & $0,0 \mathrm{~b}$ & $65,1 \mathrm{a}$ & $57,8 \mathrm{~b}$ & $57,8 \mathrm{~b}$ \\
\hline $\begin{array}{l}\text { T6) glyphosate }+ \\
\text { carfentrazone-ethyl }\end{array}$ & $\begin{array}{c}1,44+ \\
0,04\end{array}$ & $82,6 \mathrm{~b}$ & $97,2 \mathrm{a}$ & $94,4 \mathrm{a}$ & 88,6 a & $87,0 \mathrm{a}$ & $0,0 \mathrm{~b}$ & $0,0 \mathrm{~d}$ & $55,8 \mathrm{~b}$ & $55,8 \mathrm{~b}$ \\
\hline T7) Testemunha & - & $0,0 \mathrm{e}$ & $0,0 \mathrm{~d}$ & $0,0 \mathrm{e}$ & $0,0 \mathrm{~d}$ & $0,0 \mathrm{~d}$ & $57,4 \mathrm{a}$ & $51,8 \mathrm{ab}$ & $54,2 \mathrm{~b}$ & $54,2 \mathrm{~b}$ \\
\hline $\mathrm{F}$ & - & $450,08^{*}$ & $99,57 *$ & $154,11 *$ & $251,65^{*}$ & $59,53 *$ & $125,35^{*}$ & $23,68 *$ & $201,22 *$ & $153,31 *$ \\
\hline C.V. (\%) & - & 8,75 & 14,14 & 11,98 & 8,99 & 17,45 & 24,92 & 27,15 & 7,16 & 8,19 \\
\hline DMS (5\%) & - & 8,25 & 17,20 & 13,44 & 9,95 & 19,02 & 12,16 & 23,17 & 7,52 & 8,65 \\
\hline
\end{tabular}

\footnotetext{
${ }^{/ 1}$ Dias Após a Aplicação

* Valores seguidos de mesma letra na mesma coluna não diferem entre si pelo teste de Tukey a 5\% de probabilidade. ${ }^{\text {Ns }}=$ Não significativo.
}

Os herbicidas glyphosate e sulfosate, apesar de não terem apresentado seletividade aceitável, ainda assim foram os produtos de maior tolerância para coroa-decristo; no Experimento 1, uma vez que, diferentes dos demais tratamentos, até os 14 DAA os danos nas folhas foram relativamente baixos (Figura 1). A característica do teor de clorofila na folhas (Tabela 1) também indicou a maior tolerância da coroa-de-cristo ao glyphosate e sulfosate aplicados isoladamente, com valores significativamente semelhantes à testemunha dos 14 aos 21 DAA, assim como respectivamente superiores à mesma, a partir dos 28 DAA. A condição de superioridade no teor de clorofila das folhas, indicada para os tratamentos com glyphosate e sulfosate, mediante avaliação com clorofilômetro, possivelmente possa ser justificada pelo processo e/ou período de desintoxicação do efeito herbicida pelas plantas de coroa-de-cristo, visto que as folhas do ápice não caíram, mas foram intensificando a sua coloração verde com o transcorrer das épocas de avaliações.

Os herbicidas glyphosate e sulfosate são inibidores da enzima enol-piruvil shiquimato fosfato sintase (EPSPs), presente na rota da síntese dos aminoácidos aromáticos fenilalanina, tirosina e triptofano, assim como de algumas vitaminas (A e E), hormônios (auxina e etileno), alcalóides, lignina, antocianina entre outros compostos secundários das plantas. As espécies vegetais apresentam diferentes graus de metabolização dos inibidores de EPSPs, uma vez que também não penetram em caules lenhosos, sem epiderme verde, de árvores e viderias, frutíferas, etc. (DEVINE et al. 1993; FRANZ et al., 1997; KRUSE et al., 2000; VIDAL \& MEROTTO JÚNIOR, 2001; KISSMANN, 2003; CHRISTOFFOLETI, 2004).

Todos os tratamentos do Experimento 1, com exceção do paraquat, apresentaram novas rebrotas de folhas aos 35 DAA, sendo que os níveis de clorofila nas mesmas foram significativamente semelhantes aos encontrados nas testemunhas. Entretanto, mesmo assim, nas doses estudadas, os herbicidas do Experimento 1 não indicaram viabilidade para utilização comercial devido ao elevado nível de fitointoxicação provocado às plantas de coroa-de-cristo em relação à preservação dos aspectos visuais.

No Experimento 2 (Tabela 2), observa-se que os herbicidas "latifolicidas" carfentrazone-ethyl $(0,12 \mathrm{~kg}$.ha1), 2,4-D (1,3 kg.ha-1) e lactofen $\left(0,18 \mathrm{~kg} \cdot \mathrm{ha}^{-1}\right)$ não apresentaram viabiabilidade seletiva para coroa-de-cristo (Figura 2), pois promoveram danos visuais intensos de fitointoxicação a partir dos 14 DAA, caracterizados pelo necrosamento de flores, folhas e ápices das plantas, seguidos de queda das referidas estruturas. O herbicida carfentrazone-ethyl causou a morte das plantas de coroa- 


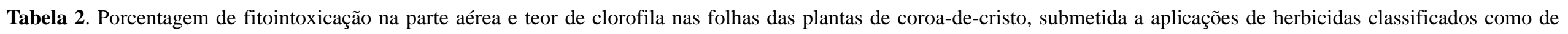
"latifoliadicidas" (Experimento 2). ESAPP/Paraguaçu Paulista-SP, 2001/2002.

\begin{tabular}{|c|c|c|c|c|c|c|c|c|c|c|}
\hline \multirow{2}{*}{ Tratamentos } & \multirow{2}{*}{$\frac{\text { Doses }}{\text { kg i.a .ha-1 }}$} & \multicolumn{5}{|c|}{ Fỉointoxicação (\%) } & \multicolumn{4}{|c|}{ Teor de Corofila $\left(\mu \mathrm{g} \mathrm{dm}^{-2}\right)$} \\
\hline & & $7 \mathrm{DAA}^{4}$ & 14 DAA & 21 DAA & $28 \mathrm{DAA}$ & 35 DAA & $14 \mathrm{DAA}$ & $21 \mathrm{DAA}$ & 28 DAA & 35 DAA \\
\hline $\begin{array}{c}\text { T1) carfentrazone- } \\
\text { ethyl }\end{array}$ & 0,12 & 88,6 a & 100,0 a & 99,0 a & 97,2 a & 97,6 a & $0,0 \mathrm{~b}$ & $0,0 \mathrm{~b}$ & $0,0 \mathrm{~b}$ & $0,0 \mathrm{c}$ \\
\hline T2) 2,4-D & 1,30 & $24,0 \mathrm{~b}$ & $60,0 \mathrm{~b}$ & $74,4 b$ & $90,0 \mathrm{~b}$ & $90,0 \mathrm{~b}$ & $61,8 \mathrm{a}$ & 57,0 a & $0,0 \mathrm{~b}$ & $0,0 \mathrm{c}$ \\
\hline T3) iscox aflutole & 0,06 & 8,0 de & 5,6 ef & 5,4 e & $4,6 \mathrm{de}$ & 3,8 de & $49,2 \mathrm{a}$ & 53,4 a & 58,3 a & $58,3 \mathrm{ab}$ \\
\hline T4) ricosulfuron & 0,06 & $9,8 \mathrm{~d}$ & $10,8 \mathrm{de}$ & $2,0 \mathrm{f}$ & $2,8 \mathrm{de}$ & $4,4 \mathrm{cde}$ & 56,2 a & 55,5 a & 57,7 a & $57,7 \mathrm{ab}$ \\
\hline $\begin{array}{c}\text { T5) metsulfuron- } \\
\text { m ethyl }\end{array}$ & 0,0024 & $2,6 \mathrm{gh}$ & $1,8 \mathrm{fg}$ & $0,0 \mathrm{f}$ & $0,0 \mathrm{e}$ & 0,0 e & 59,3 a & 54,3 a & 52,4 a & 52,4 b \\
\hline T6) 1actofen & 0,18 & $2,0 \quad h$ & $62,0 \mathrm{~b}$ & $64,2 \mathrm{c}$ & $86,2 \mathrm{~b}$ & $92,2 \mathrm{~b}$ & 57,5 a & 59,7 a & $55,8 \mathrm{a}$ & 60,7 a \\
\hline $\begin{array}{l}\text { T7) chlorimuron- } \\
\text { ethyl }\end{array}$ & 0,02 & $18,6 \mathrm{c}$ & $38,6 \mathrm{c}$ & $14,2 \mathrm{~d}$ & $16,4 \mathrm{c}$ & $7,0 \mathrm{~cd}$ & 58,8 a & 54,8 a & 58,3 a & $58,3 \mathrm{ab}$ \\
\hline T8) bentazon & 1,25 & 5,6 ef & $14,0 \mathrm{~d}$ & $15,0 \mathrm{~d}$ & $5,4 \mathrm{~d}$ & $9,2 \mathrm{c}$ & 51,5 a & 59,4 a & 59,2 a & $58,2 \mathrm{ab}$ \\
\hline T9) testem unha & - & $0,0 \quad \mathrm{~h}$ & $0,0 \mathrm{~h}$ & $0,0 \mathrm{f}$ & 0,0 e & $0,0 \quad \mathrm{e}$ & 57,4 a & $51,8 \mathrm{a}$ & 54,2 a & $54,2 \mathrm{ab}$ \\
\hline $\mathrm{F}$ & - & $1670,35^{*}$ & $973,81 *$ & $3020,16^{*}$ & $1712,12^{*}$ & $1918,38 *$ & $45,82 *$ & $31,21 *$ & $114,51 *$ & $235,13^{*}$ \\
\hline C.V. $(\%)$ & - & 8,58 & 7,69 & 5,08 & 6,99 & 6,76 & 12,65 & 15,16 & 11,89 & 8,31 \\
\hline $\operatorname{DMS}(5 \%)$ & - & 3,19 & 5,26 & 3,25 & 4,94 & 4,80 & 13,34 & 15,78 & 10,99 & 7,75 \\
\hline
\end{tabular}

${ }^{11}$ Dias Após a Aplicação

* Valores seguidos de mesma letra na mesma coluna não diferem entre si pelo teste de Tukey a $5 \%$ de probabilidade. ${ }^{\text {Ns }}=$ Não significativo. 

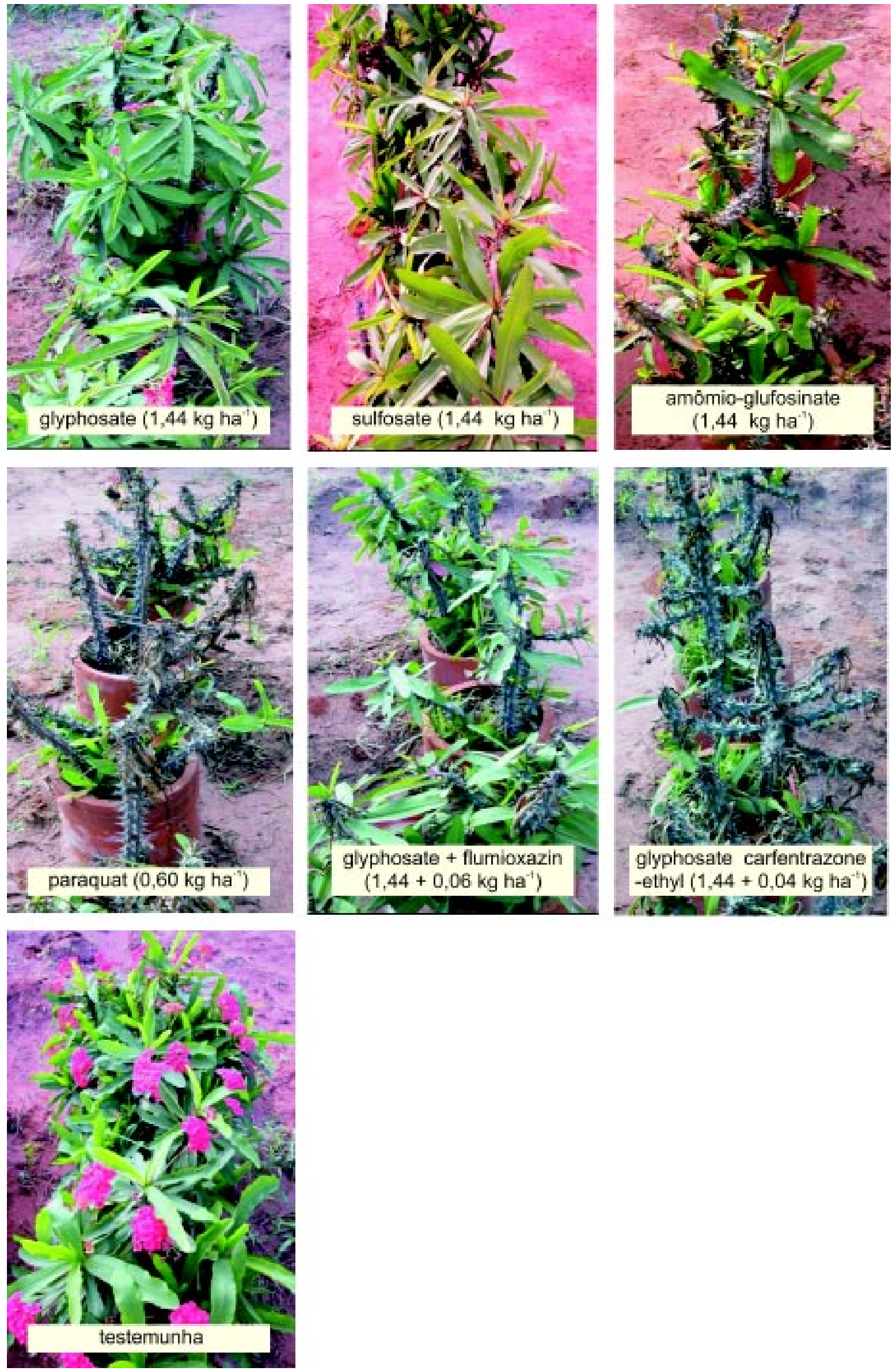

Figura 1. Danos visíveis de fitointoxicação na parte aérea das plantas de coroa-de-cristo aos 14 DAA, submetida aos efeitos de herbicidas classificados como de "ação total" (Experimento 1). ESAPP/Paraguaçu Paulista-SP, 2001/2002. 

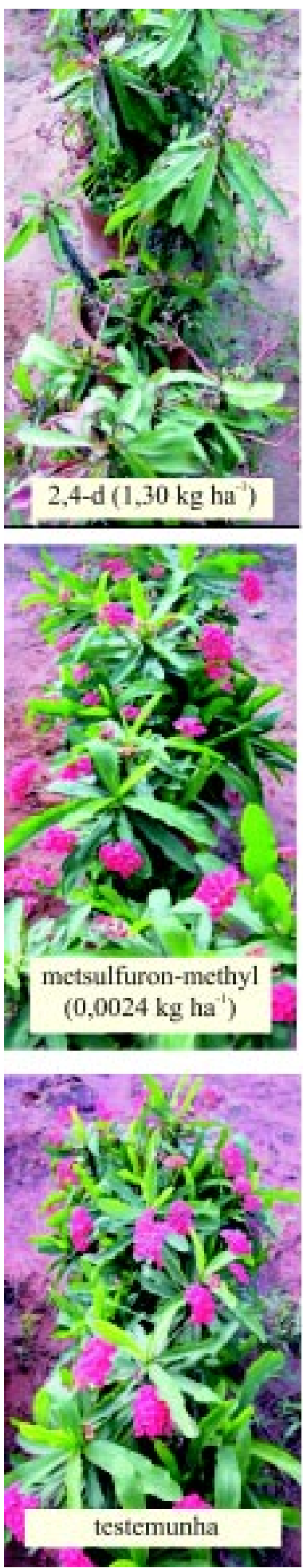
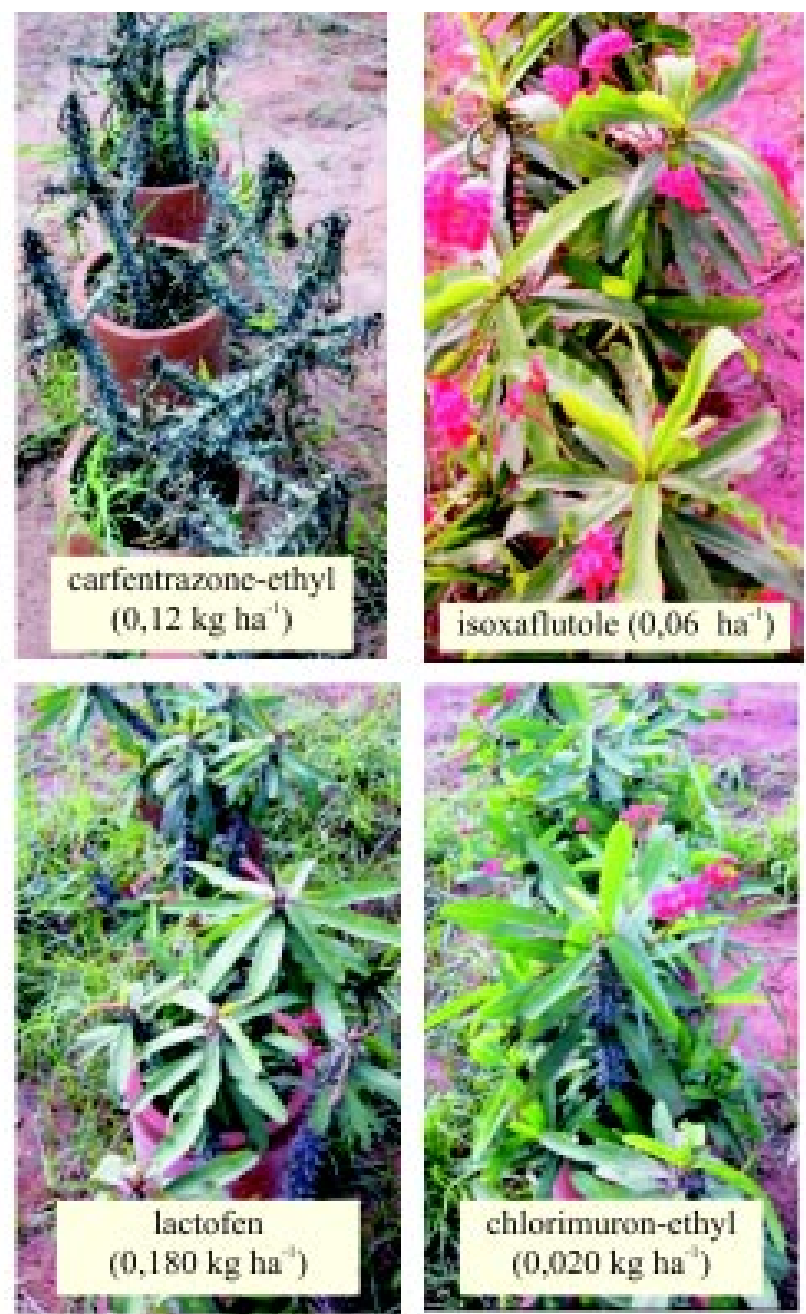
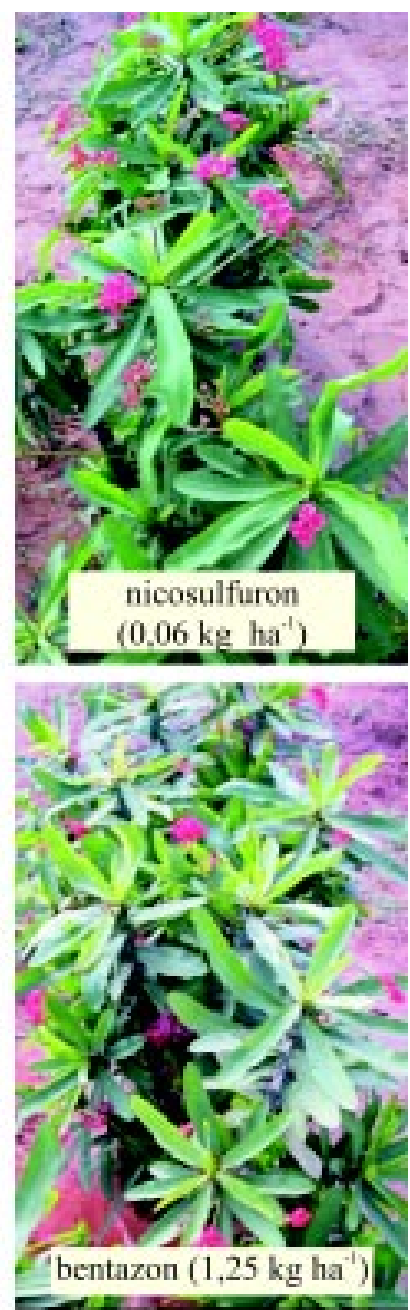

Figura 2. Danos visíveis de fitointoxicação na parte aérea das plantas de coroa-de-cristo aos 14 DAA, submetida aos efeitos de herbicidas classificados como "latifolicidas" (Experimento 2). ESAPP/Paraguaçu Paulista-SP, 2001/2002.

de-cristo a partir dos 7 DAA. Entre os 7 e 14 DAA, o herbicida 2,4-D resultou em acentuada epinastia das inflorescências e morte das flores, seguida de avermelhamento e enrolamento de várias folhas do ápice, e inclinamento para baixo das folhas, formando na parte aérea da coroa-de-cristo um aspecto de "guarda-chuva semi-aberto". Aos 28 DAA, as quedas das estruturas flores e folhas atingiram nível pleno para o 2,4-D, não havendo formação de rebrotas até 35 DAA. Para o lactofen, o nível de danos nas gemas apicais, inflorescências e folhas foi intenso a partir dos 14 DAA, com avermelhamento e manchas cloróticas nas folhas, que interferiram no teor de clorofila até os 35 DAA, mas causaram efeito "guardachuva semi-aberto" e elevada supressão de desenvolvimento da parte aérea.

De forma contrária, ainda no Experimento 2, o tratamento com metsulfuron-methyl $\left(0,0024 \mathrm{~kg} \cdot \mathrm{ha}^{-1}\right)$ apresentou ampla seletividade à coroa-de-cristo, sem reduzir a qualidade visual e o teor de clorofila das folhas, e, principalmente, o desenvolvimento das inflorescências 
Tabela 3. Porcentagem de fitointoxicação na parte aérea e teor de clorofila nas folhas das plantas de coroa-de-cristo, submetida a aplicações de herbicidas classificados como de "graminicidas" (Experimento 3). ESAPP/Paraguaçu PaulistaSP, 2001/2002.

\begin{tabular}{|c|c|c|c|c|c|c|c|c|}
\hline \multirow[t]{2}{*}{ Tratamentos } & \multirow{2}{*}{$\begin{array}{c}\text { Dosagem } \\
\text { kg i.a. ha-1 }\end{array}$} & \multicolumn{5}{|c|}{ Fütointoxicação (\%) } & \multicolumn{2}{|c|}{$\begin{array}{l}\text { Teor de Corofila } \\
\quad\left(\mu g \mathrm{dm}^{-2}\right)\end{array}$} \\
\hline & & $\begin{array}{c}7 \\
\text { DAA }^{11}\end{array}$ & $\begin{array}{c}14 \\
\text { DAA }\end{array}$ & $\begin{array}{c}21 \\
\text { DAA }\end{array}$ & $\begin{array}{c}28 \\
\text { DAA }\end{array}$ & $\begin{array}{c}35 \\
\text { DAA }\end{array}$ & $\begin{array}{c}14 \\
\text { DAA }\end{array}$ & $28 \mathrm{DAA}$ \\
\hline $\begin{array}{l}\text { T1) fluazifop-p- } \\
\text { buty1 }\end{array}$ & 0,250 & $13,2 \mathrm{~b}$ & $13,0 \mathrm{a}$ & $10,0 \mathrm{a}$ & $8,8 \mathrm{a}$ & $5,0 \mathrm{a}$ & 49,6 & $61,8 \mathrm{ab}$ \\
\hline T2) sethoxydim & 1,250 & 18,4 a & $7,2 \mathrm{c}$ & $7,2 \mathrm{~b}$ & $5,8 \mathrm{~b}$ & $3,2 \mathrm{a}$ & 55,8 & $65,8 \mathrm{ab}$ \\
\hline $\begin{array}{l}\text { T3) haloxyfop- } \\
\text { methyl }\end{array}$ & 0,060 & $1,4 \mathrm{~d}$ & $1,2 \mathrm{de}$ & $0,0 \mathrm{~d}$ & $0,0 \mathrm{~d}$ & $0,0 \mathrm{~b}$ & 60,2 & $65,8 \mathrm{ab}$ \\
\hline $\begin{array}{l}\text { T4) clethodim+ } \\
\text { fenoxaprop-p-ethyl }\end{array}$ & 0,110 & $2,4 \mathrm{~cd}$ & $8,8 \mathrm{bc}$ & $2,2 \mathrm{c}$ & $0,0 \mathrm{~d}$ & $0,0 \mathrm{~b}$ & 59,8 & $68,4 a$ \\
\hline $\begin{array}{l}\text { T5) quizalofop-p- } \\
\text { ethy1 }\end{array}$ & 0,100 & $2,2 \mathrm{~cd}$ & $8,8 \mathrm{bc}$ & $1,6 \mathrm{~cd}$ & $0,0 \mathrm{~d}$ & $0,0 \mathrm{~b}$ & 54,6 & $55,5 \mathrm{c}$ \\
\hline T0) clethodim & 0,120 & $10,8 \mathrm{~b}$ & $9,2 \mathrm{bc}$ & $3,0 \mathrm{c}$ & $0,0 \mathrm{~d}$ & $0,0 \mathrm{~b}$ & 57,5 & $58,3 \mathrm{bc}$ \\
\hline T7) propacuizafop & 0,125 & $9,8 \mathrm{~b}$ & $10,8 \mathrm{ab}$ & $2,0 \mathrm{c}$ & $2,8 \mathrm{c}$ & $4,4 \mathrm{a}$ & 56,2 & $57,7 \mathrm{bc}$ \\
\hline T8) tepraloxydim & 0,400 & $5,2 \mathrm{c}$ & $2,8 \mathrm{~d}$ & $1,4 \mathrm{~cd}$ & $0,4 d$ & $0,0 \mathrm{~b}$ & 60,2 & 56,6 bc \\
\hline T9) butroxydim & 0,100 & $2,6 \mathrm{~cd}$ & $3,4 \mathrm{~d}$ & $0,0 \mathrm{~d}$ & $0,0 \mathrm{~d}$ & $0,0 \mathrm{~b}$ & 58,8 & $54,3 \mathrm{c}$ \\
\hline T10) testemurha & - & $0,0 \mathrm{~d}$ & $0,0 \mathrm{e}$ & $0,0 \mathrm{~d}$ & $0,0 \mathrm{~d}$ & $0,0 \mathrm{~b}$ & 57,4 & $54,2 \mathrm{c}$ \\
\hline $\mathrm{F}$ & - & $58,99 *$ & $70,33 *$ & $73,21 *$ & $82,76^{*}$ & $21,71^{*}$ & $1,17^{\mathrm{K}}$ & $7,23^{*}$ \\
\hline C.V. (\%) & - & 26,96 & 17,92 & 31,65 & 43,03 & 79,01 & 11,75 & 7,28 \\
\hline $\operatorname{DMS}(5 \%)$ & - & 3,79 & 2,49 & 1,85 & 1,63 & 1,26 & 14,28 & 9,28 \\
\hline
\end{tabular}

\footnotetext{
${ }^{11}$ Dias Após a Aplicação

* Valores seguidos de mesma letra na mesma coluna não diferem entre si pelo teste de Tukey a $5 \%$ de probabilidade. ${ }^{\text {Ns }}=$ Não significativo.
}

(Tabela 2). Os herbicidas isoxaflutole $\left(0,06 \mathrm{~kg} \mathrm{ha}^{-1}\right)$, nicosulfuron $\left(0,06 \mathrm{~kg} \cdot \mathrm{ha}^{-1}\right)$, chlorimuron-ethyl $(0,02 \mathrm{~kg} \cdot \mathrm{ha}$ $\left.{ }^{1}\right)$ e bentazon $\left(0,18 \mathrm{~kg} \cdot \mathrm{ha}^{-1}\right)$, na respectiva ordem, também apresentaram aspectos positivos com relação à seletividade para coroa-de-cristo, resultando em média uma baixa preservação dos aspectos visuais das inflorescências e do pouco comprometimento da parte vegetativa, na qual os danos e a leve supressão do desenvolvimento das folhas não causaram prejuízos em relação à qualidade dos aspectos visuais (Figura 2). Nesse contexto, os herbicidas isoxaflutole, nicosulfuron, chlorimuron-ethyl e bentazon, nas referidas doses estudadas, poderiam ser adequadamente utilizados no manejo de plantas daninhas estando a coroa-de-cristo em estádio de não florescimento.

Os herbicidas metsulfuron-methyl, nicosulfuron e chlorimuron-ethyl pertencem ao grupo químico das sulfoniluréias, cujo mecanismo de ação é inibidor da enzima acetolactato sintase (ALS), responsável pela síntese de aminoácidos alifáticos de cadeia lateral valina, leucina e isoleucina. É considerado um importante grupo de produtos devido à utilização de doses reduzidas, grande espectro de plantas daninhas controladas e perfil toxicológico bastante favorável, visto que agem em uma enzima ausente em mamíferos (CHRISTOFFOLETI, 1997; RODRIGUES \& ALMEIDA, 1998; VIDAL \& MEROTTO JÚNIOR, 2001). Diferente das sulfoniluréias, o bentazon atua no cloroplasto, inibindo o transporte de elétrons no fotossistema II (VIDAL, 1997; RODRIGUES \& ALMEIDA, 1998).

Para os herbicidas classificados como "graminicidas", avaliados no Experimento 3, todos os tratamentos apresentaram viabilidade seletiva à coroa-decristo (Tabela 3). Manifestações de danos mais intensos foram constatadas somente nas flores para o herbicida fluazifop-p-butil $\left(0,25 \mathrm{~kg} \cdot \mathrm{ha}^{-1}\right)$, o qual não preservou os aspectos visuais das referidas estruturas, e de forma menos representativa para sexthoxydim $\left(1,25 \mathrm{~kg} \cdot \mathrm{ha}^{-1}\right) \mathrm{e}$ propaquizafop $\left(0,125 \mathrm{~kg} \cdot \mathrm{ha}^{-1}\right)$, cujas injúrias causaram apenas redução parcial da intensidade de florescimento das plantas (Figura 3). De forma geral, nenhum dos herbicidas graminicidas causou injúrias comprometedoras nas estruturas foliares e/ou na supressão do desenvolvimento vegetativo das plantas, caracterizandose apenas por leves e irregulares manchas cloróticas, pontuações esbranquiçadas e/ou encarquilhamento 

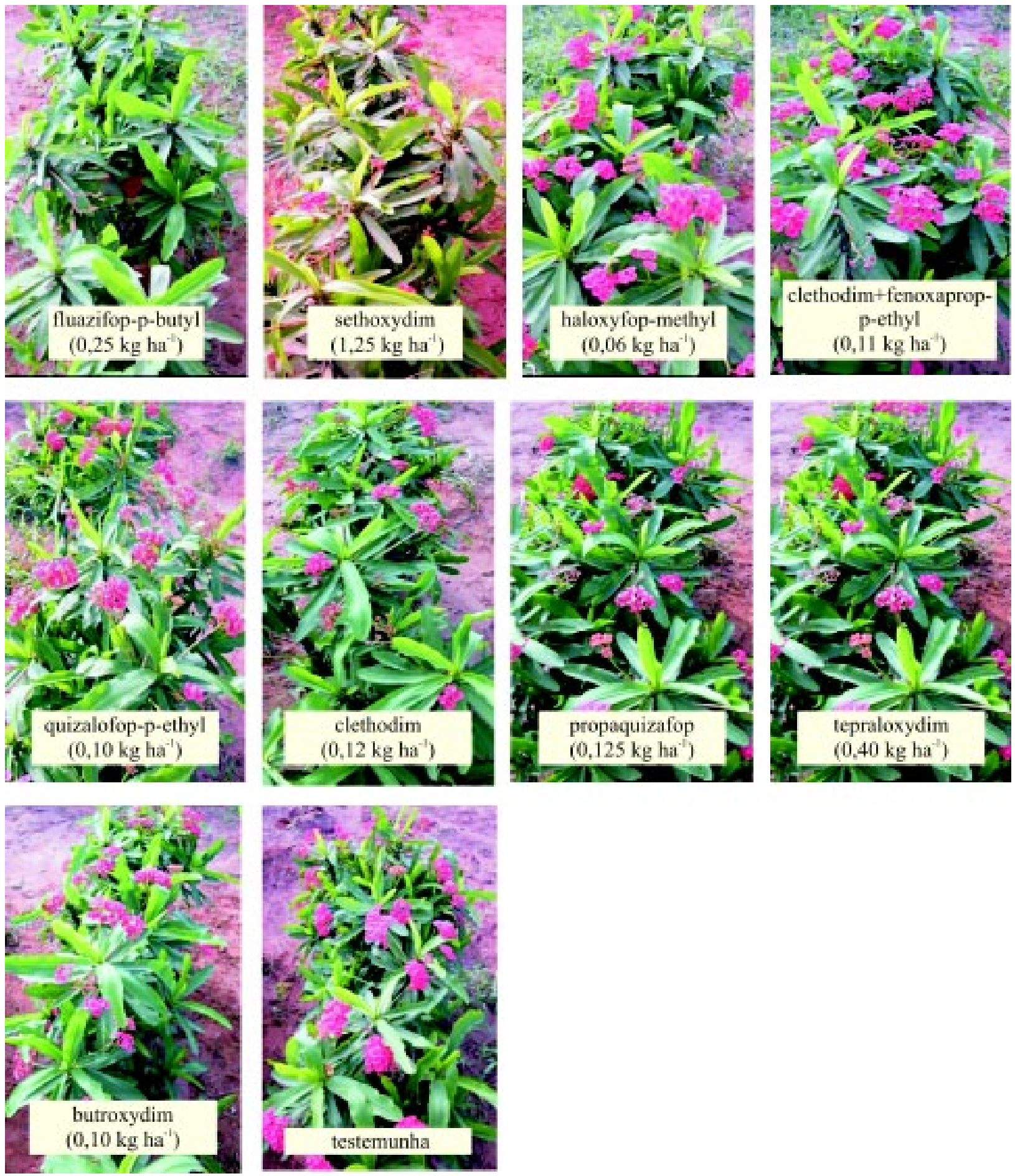

Figura 3. Danos visíveis de fitointoxicação na parte aérea das plantas de coroa-de-cristo aos 14 DAA, submetida aos efeitos de herbicidas classificados como “graminicidas” (Experimento 3). ESAPP/Paraguaçu Paulista-SP, $2001 / 2002$.

parcial da região mediana do limbo foliar. Os resultados sugerem que os herbicidas fluazifop-p-butil, sexthoxydim e propaquizafop poderiam ser utilizados apenas no estádio de desenvolvimento vegetativo da coroa-de-cristo, e os herbicidas haloxyfop-methyl, clethodim + fenoxaprop-pethyl, quizalofop-p-ethyl, clethodim, tepraloxydim e butroxydim com as planta em estádio de florescimento pleno e/ou somente na presença das folhas.

Os herbicidas estudados no Experimento 3 são classificados como inibidores da enzima acetil-Coenzima A carboxilase (ACCase), a qual é responsável por participar na síntese de malonil-CoA, bloqueando a reação inicial da rota metabólica da síntese de lipídios na plantas (VIDAL,
1997). Nas últimas duas décadas, os herbicidas desse grupo têm assumido grande importância, por possibilitarem o controle seletivo em pós-emergência de espécies da família poaceae em culturas dicotiledôneas, e em alguns casos, também em culturas monocotiledôneas (KUK et al., 2000).

Pode-se concluir que houve viabilidade do uso do manejo químico para o controle de plantas daninhas em localidades onde se encontra a espécie coroa-de-cristo $(E$. splendens). Entretanto, deve-se considerar que a escolha do herbicida estará em função do seu potencial seletivo, e deverá ser caracterizada pela preservação e/ou manutenção da qualidade dos aspectos de jardinagem para essa espécie ornamental. Nesse sentido, também é de fundamental 
importância o estádio fenológico das plantas coroa-decristo no momento da aplicação, para que o sucesso da adoção dessa tecnologia seja pleno.

\section{REFERÊNCIASBIBLIOGRÁFICAS}

BIACHINI, F.; PANTANO, A. C. Tudo Verde. Guia das Plantas e Flores. São Paulo: Melhoramentos. p.50, 1994. BLOSSFELD, H. Jardinagem. São Paulo: Melhoramentos, 1965. 417p.

CHRISTOFFOLETI, P.J. Resistência de plantas daninhas aos herbicidas. In: I SIMPÓSIO SOBRE HERBICIDAS E PLANTAS DANINHAS, 1.,1997. Dourados. Resumos. Dourados: Embrapa-CPAO, p.75-94, 1997.

CHRISTOFFOLETI, P.J.; LÓPEZ-OVERJERO, R.F.; CARVALHO, J.C. Aspectos de resistência de plantas daninhas a herbicidas. CHRISTOFFOLETI, P. J. (Coord.). 2. ed Londrina: Associação Brasileira de Ação a Resistência de Plantas Daninhas aos Herbicidas (HRAC-BR), 2004. 100p.

DEVINE, M.; DUKE, S.O; FEDTKE, C. Physiology of herbicide action. Englewood cliffs, PTR Prenctice Hall, 1993, 441p.

EWRC(EUROPEANWEEDRESEARCHCOUNCIL). Report of the 3rd and 4Th Meetings of ERWC - Committee of Methods In Weed Research. Weed Research, v.4, p.88, 1971.

FRANZ; J.E.; MAO, M.K.; SIKORSKI, J.A. Glyphosate: a unique global herbicide. Washington, ACS monograph, 1997. 653p.

IBGE - Cidades. Instituto Brasileiro de Geografia e Estatística. Coordenadas Geográficas das Cidades, 2004. Disponível em: <http://www.ibge.gov.br/home/presidencia/noticias / notavisualiza.php?idnota=3> Acesso em: 3 jan. 2004 .
KISSMANN, K.G. Resistência de plantas daninhas a herbicidas. Disponível em: http://www.hrac-br.com.br/ arquivos/texto_resistencia_herbicidas.doc 2003

KRUSE, N.D.; TREZZI, M.M.; VIDAL, R.A. Herbicidas inibidores de EPSPs: Revisão de literatura. Revista Brasileira de Herbicidas, Brasília, v.1., p.139-146, 2000.

KUK, Y.I.; BURGOS, N.R.; TALBERT, R.E. Cross and multiple resistence of diclofop-resistant Lolium spp. Weed Science, v.48, n.4, p.412-419, 2000.

LORENZI, H. Plantas daninhas do Brasil: terrestres, aquáticas, parasitas e tóxicas. 3. ed. Nova Odessa: Instituto Plantarum, p.205, 2000.

LORENZI, H.; SOUZA, H. M. Plantas ornamentais do Brasil: arbustivas, herbáceas e trepadeiras. 3. ed. Nova Odessa: Instituto Plantarum, p.511-512, 2001.

RODRIGUES, B.N.; ALMEIDA, F.S. Guia de Herbicidas. 4. ed. Londrina, ed. dos autores, 1998. 648p.

RURALNEWS. Cercas vivas. Jardinagem e Paisagismo, 2004. Disponível em: < http://www.ruralnews.com.br/ default. asp?cod=57> Acesso em: 3 jan. 2004.

SOCIEDADE BRASILEIRADACIÊNCIADAS PLANTAS DANINHAS. Procedimentos para instalação, avaliação e análise de experimentos com herbicidas. Londrina: SBCPD, 1995. 42p.

VIDAL, R.A. Herbicidas: mecanismo de ação e resistência de plantas. Porto Alegre: ed. do autor, 1997. 165p.

VIDAL, R.A.; MEROTTO JÚNIOR, A. Herbicidologia. 1. ed. Porto alegre: s.n., 2001.

ZIMBER, G. Jardins de hoje. São Paulo: s.n, 1946. 139p. 